\title{
NOTE ON SIMPLE INTEGRAL EXTENSION DOMAINS AND MAXIMAL CHAINS OF PRIME IDEALS
}

\author{
L. J. RATLIFF, JR. ${ }^{1}$
}

\begin{abstract}
It is shown that if $R$ is a semi-local (Noetherian) domain, then there exists a simple integral extension domain $R[e]$ of $R$ such that there exists a maximal chain of prime ideals of length $n$ in some integral extension domain of $R$ if and only if there exists a maximal chain of prime ideals of length $n$ in $R[e]$. An interesting existence theorem on a certain type of height one prime ideals in $R[X]$ follows.
\end{abstract}

1. Introduction. All rings in this paper are assumed to be commutative with identity and the undefined terminology is the same as that in [3].

In some personal correspondence with Steve McAdam in 1974 the following question arose: if $R$ is a local domain, then does there exist a simple integral extension domain $R[e]$ of $R$ such that there exists a maximal chain of prime ideals of length $n$ in some integral extension domain of $R$ (if and) only if there exists such a chain of prime ideals in $R[e]$ ? (See [8, (15.4.2)].) This question is of some interest, since if the answer is no, then the Upper Conjecture (see (3.2) below) is false, and so a number of the other catenary chain conjectures are also false (see [8, p. 44]). However, we show in (3.1) that this question has a strong affirmative answer, and so we thereby lend at least some support to the Upper Conjecture.

The proof of (3.1) depends on two quite recent results which are summarized in $\$ 2$, and therein we also give the relevant definitions and notation that are needed in $\$ 3$. $\$ 3$ contains a proof of the theorem together with a few of its corollaries and related results. Among these, we show that the theorem also holds for arbitrary integral extensions domains of $R$ and that it implies the existence of a certain type of height one prime ideal in $R[X]$. A final result, (3.6), shows that certain other questions concerning maximal chains of prime ideals in integral extension domains of $R$ have a solution in simple integral extension domains of $R$ if they have a solution at all.

2. Definitions and known results. We begin with two definitions.

(2.1) Definition. A chain of prime ideals $P_{0} \subset P_{1} \subset \cdots \subset P_{n}$ in a ring $A$

Received by the editors September 20, 1978.

AMS (MOS) subject classifications (1970). Primary 13A15, 13B20, 13C15; Secondary 13B25.

Key words and phrases. Integral extension ring, maximal chain of prime ideals, polynomial ring, semi-local ring, simple extension ring, Upper Conjecture.

${ }^{1}$ Research on this paper was supported in part by the National Science Foundation Grant MCS77-00951, A01. 
is said to be a maximal chain of prime ideals in $A$ in case $P_{0}$ is a minimal prime ideal, $P_{n}$ is a maximal ideal, and there are no prime ideals in $A$ properly between $P_{i-1}$ and $P_{i}$ for $i=1, \ldots, n$. The length of the chain is $n$.

(2.2) Definition. For a ring $A, c(A)$ (resp., $u c(A)$ ) denotes the set of lengths of maximal chains of prime ideals in $A$ (resp., in arbitrary integral extension domains of $A / z$ with $z$ varying over the minimal prime ideals in $A$ ). When $P \in \operatorname{Spec} A$ we let $c(P)$ and $u c(P)$ denote $c\left(A_{P}\right)$ and $u c\left(A_{P}\right)$, respectively.

It is known [3, Example 2, pp. 203-205] that, even when $A$ is a local domain, $c(A)$ may have more than one element and $c(A)$ may be a proper subset of $u c(A)$. However, in all known examples of this it is at least true that there exists a simple integral extension domain $A[b]$ of $A$ such that $c(A[b])=$ $u c(A)$, and this led to the question mentioned in the Introduction.

At this point it should be noted that if in this question we did not require the ring $R[e]$ to be an integral domain then the answer is readily seen to be yes; for if $i \in u c(R)$, then there exists a simple integral extension domain $R\left[b_{i}\right]$ of $R$ such that $i \in c\left(R\left[b_{i}\right]\right)$, by [1, Theorem 1.10], so there exists a monic polynomial $f_{i}(X)$ in $R[X]$ contained in $\operatorname{Ker}\left(R[X] \rightarrow R\left[b_{i}\right]\right)$, and so on letting $f(X)=\pi\left\{f_{i}(X) ; i \in u c(R)\right\}$ we have

$$
c(R[X] / f(X) R[X])=u c(R) .
$$

The following remark lists some known (and for the most part easily proved) facts about $c(A)$ and $u c(A)$ that will be needed in $\$ 3$.

(2.3) REMARK. The following statements hold for a ring $A$ :

(2.3.1) $c(A) \subseteq u c(A)$.

(2.3.2) $c(A)=\cup\{c(M) ; M$ is a maximal ideal in $A\}$.

(2.3.3) $u c(A)=\cup\{u c(M) ; M$ is a maximal ideal in $A\}$.

(2.3.4) If $A$ is an integral domain and $B$ is an integral extension domain of $A$, then $c(A) \subseteq c(B)$ and $u c(B)=u c(A)$ by the Going Up Theorem.

(2.3.5) If $A$ is local and $B$ is a quasi-local integral extension ring of $A$ such that minimal prime ideals in $B$ lie over minimal prime ideals in $A$, then $c(A)=c(B)$ by $[4,(3.4)]$.

This section will be closed with a notational convention and by stating the main result in two recent papers that are needed in the proof of (3.1).

(2.4) Notation. $A^{\prime}$ denotes the integral closure of a ring $A$ in its total quotient ring.

(2.5) [7, (2.4)]. If $R$ is a semi-local domain, then there exists a finite integral extension domain $A$ of $R$ such that, for each integral extension domain $B$ of $A$ (including $A$ ) and for each maximal ideal $P$ in $B, u c(P)=c(P)=u c\left(M^{\prime}\right)$ for some maximal ideal $M^{\prime}$ in $R^{\prime}$ (2.4). (The ideal $M^{\prime}$ is the ideal $P^{\prime} \cap R^{\prime}$ where $P^{\prime}$ is any of the maximal ideals in $B^{\prime}$ that lies over $P$.)

(2.6) [9, (2.3), (2.18), and (2.19)]. If $R \subseteq A$ are quasi-semi-local rings such that $A$ is integral over $R$, then the following statements hold:

(2.6.1) If $R / M$ is an infinite field for each maximal ideal $M$ in $R$, then 
there exist $a \in A$ such that $R[a]$ and $A$ have the same number of maximal ideals.

(2.6.2) There always exist simple integral extension rings $A[e]$ of $A$ such that $A, A[e]$, and $R[e]$ all have the same number of maximal ideals. Moreover, $e$ can be chosen such that minimal prime ideals in $A[e]$ lie over minimal prime ideals in $A$, and such that $A[e]$ is an integral domain whenever $A$ is an integral domain.

3. Simple integral extension domains and maximal chains of prime ideals. As mentioned in the introduction we give, in (3.1), a strong affirmative answer to a question that arose in 1974 concerning maximal chains of prime ideals in integral extension domains of a local domain, and then we prove a few corollaries of (3.1). (It should be noted that (3.1) is a considerable strengthening of the result in [1] mentioned preceding (2.3). However, it does not supercede that result, since it was used in the proof of (2.5), which we use below.)

(3.1) THEOREM. If $\left(R ; M_{1}, \ldots, M_{h}\right)$ is a semi-local domain, then there exists a simple integral extension domain $R[e]$ of $R$ such that $c(R[e])=u c(R)$. Moreover, the following statements hold:

(3.1.1) For each integral extension domain $B$ of $R[e]$ and for each maximal ideal $P$ in $B$ such that either: (i) $P$ is not contained in the union of the other maximal ideals in $B$, or (ii) $P$ does not contain the intersection of the other maximal ideals in $B$; we have $u c(P)=c(P)=u c\left(M^{\prime}\right)$ for some maximal ideal $M^{\prime}$ in $R^{\prime}$ (2.4).

(3.1.2) If each $R / M_{i}$ is infinite, then $e$ can be chosen such that, for each integral extension domain $B$ of $R[e]$ and for each maximal ideal $P$ in $B$, $u c(P)=c(P)=u c\left(M^{\prime}\right)$ for some maximal ideal $M^{\prime}$ in $R^{\prime}$.

Proof. By (2.5) let $A$ be a finite integral extension domain of $R$ such that, for each integral extension domain $D$ of $A$ (including $A$ ) and for each maximal ideal $P$ in $D, u c(P)=c(P)=u c\left(M^{\prime}\right)$, for some maximal ideal $M^{\prime}$ in $R^{\prime}$. By (2.6.2) let $A[e]$ be a simple integral extension domain of $A$ such that $A$, $A[e]$, and $R[e]$ all have the same number of maximal ideals.

Then for each maximal ideal $N$ in $A[e], A[e]_{N}$ is integral over $R[e]_{N \cap R[e]}$, so $c(N)=c(N \cap R[e])$, by (2.3.5). Also, $u c(N)=u c(N \cap R[e])$, by (2.3.4). Therefore by (2.3.1) and the property of $A$ we have

$$
c(N \cap R[e]) \subseteq u c(N \cap R[e])=u c(N)=c(N)=c(N \cap R[e])
$$

Thus it follows from the choice of $e$ that $c(Q)=u c(Q)$ for all maximal ideals $Q$ in $R[e]$. Therefore if $m \in u c(R)$, then $m \in u c(R[e])$, by (2.3.4), so $m \in$ $u c(Q)$ for some maximal ideal $Q$ in $R[e]$, by (2.3.3), hence $m \in c(Q)$, by the preceding sentence, and so $m \in c(R[e])$, by (2.3.2). Therefore it follows that $u c(R) \subseteq c(R[e])$, and $c(R[e]) \subseteq u c(R[e])=u c(R)$, by (2.3.1) and (2.3.4), so $u c(R)=c(R[e])$. 
To prove (3.1.1) let $B$ be an integral extension domain of $R[e]$ and let $P$ be a maximal ideal in $B$ such that (i) or (ii) holds. Then there exists $b$ in $P$ that is not in any other maximal ideal in $B$ (in case (ii) since $P$ and the intersection $I$ of the other maximal ideals in $B$ are comaximal ideals). Let $Q=P \cap R[e]$. Then $Q^{\prime}=(Q, b) R[e, b]$ is a maximal ideal and $P$ is the only maximal ideal in $B$ that lies over $Q^{\prime}$. By the choice of $e$ let $N$ be the maximal ideal in $A[e]$ that lies over $Q$. Then $N^{\prime}=(N, b) A[e, b]$ is a maximal ideal and is the only maximal ideal in $A[e, b]$ that lies over $Q^{\prime}$ (for if $N^{*}$ is a maximal ideal in $A[e, b]$ that lies over $Q^{\prime}$, then $b \in N^{*}$ and $N^{*} \cap R[e]=Q$, so necessarily $N^{*} \cap A[e]=N$, hence $\left.N^{*}=(N, b) A[e, b]\right)$. Therefore by (2.3.5) and (2.3.4) we have $c(P)=c\left(Q^{\prime}\right)=c\left(N^{\prime}\right)$ and $u c(P)=u c\left(Q^{\prime}\right)=u c\left(N^{\prime}\right)$. Also, by the property of $A$ we have $u c\left(N^{\prime}\right)=c\left(N^{\prime}\right)=u c\left(M^{\prime}\right)$ for some maximal ideal $M^{\prime}$ in $R^{\prime}$. Therefore $u c(P)=c(P)=u c\left(M^{\prime}\right)$ for some maximal ideal $M^{\prime}$ in $R^{\prime}$, and so (3.1.1) holds.

To prove (3.1.2) it suffices, by [6, (4.8.2)], to prove that $e$ can be chosen such that for each maximal ideal $Q$ in $R[e]$ we have $\left(R[e]_{Q}\right)^{\prime}$ is quasi-local and $u c(Q)=c(Q)=u c\left(M^{\prime}\right)$ for some maximal ideal $M^{\prime}$ in $R^{\prime}$. For this, by the properties of $A$ each of its finite integral extension domains has the same properties, so in particular we can assume that $A$ and $A^{\prime}$ have the same number of maximal ideals. Also, by hypothesis and (2.6.1) there exist $e \in A$ such that $A$ and $R[e]$ have the same number of maximal ideals, so the following rings all have the same number of maximal ideals: $A^{\prime}, A, R[e]$, and $R[e]^{\prime}$. Thus for each maximal ideal $Q$ in $R[e]$ we have $\left(R[e]_{Q}\right)^{\prime}$ is quasi-local. Also, it was shown in the second paragraph of this proof that $u c(Q)=c(Q)$ $=c(N)$, where $N$ is the maximal ideal in $A$ that lies over $Q$, and $c(N)=$ $u c\left(M^{\prime}\right)$ for some maximal ideal $M^{\prime}$ in $R^{\prime}$ (by the first sentence of this proof). Therefore (3.1.2) holds. Q.E.D.

I do not know if $e$ can be chosen such that the conclusion of (3.1.2) holds when some $R / M_{i}$ is finite. (It is known [9, (2.16)] that if some $R / M_{i}$ is finite, then the conclusion of (2.6.1) may not hold.)

At this point we note that (3.1) lends some support to the following conjecture.

(3.2) Upper Conjecture. If $(R, M)$ is a local domain and there exists a maximal chain of prime ideals of length $n+1>2$ in $R[X]_{(M, X)}$, then there exists a maximal chain of prime ideals of length $n$ in $R$.

It is known [6, (4.10.3)] that (3.2) is equivalent to: if $R$ is a local domain such that either altitude $R=1$ or altitude $R>1$ and there does not exist a height one maximal ideal in $R^{\prime}$, then $c(R)=u c(R)$. Thus the Upper Conjecture asserts that a very strong form of the question mentioned in $\$ 1$ holds. (3.1) gave a strong affirmative answer to this question, but not as strong as (3.2).

Two comments are given in (3.3) that should be noted in regard to (3.1). The second of these shows that every semi-local domain has a finite integral 
extension domain $S$ such that the ring $S[e]$ (as given for $S$ by (3.1)) is contained in $S^{\prime}$. It would be of some importance if it could be shown that every semi-local domain has this property of $S$.

(3.3) REMARK. With the notation of (3.1) the following statements hold:

(3.3.1) If $R$ is integrally closed, then $R[e]$ is a free $R$-algebra.

(3.3.2) Let $S=R+J$ with $J$ the Jacobson radical of $R[e]$. Then $S$ and $R$ have the same number of maximal ideals, $S \subseteq R[e] \subseteq S^{\prime}$, and $u c(S)=$ $c(R[e])$.

PROOF. (3.3.1).If $R$ is integrally closed, then $\operatorname{Ker}(R[X] \rightarrow R[e])$ is a principal ideal by the Division Algorithm, so $R[e]$ is free over $R$.

(3.3.2). The maximal ideals in $S$ are the ideals $(J, M) S$ with $M$ a maximal ideal in $R$, so $S$ and $R$ have the same number of maximal ideals. Also, $S \subseteq R[e] \subseteq S^{\prime}$, since $e$ is integral over $R$ and $S$ and $R[e]$ have the same quotient field. Finally, $u c(S)=u c(R)=c(R[e])$ by (2.3.4) and (3.1). Q.E.D.

(3.4) extends most of (3.1) to arbitrary integral extension domains of a semi-local domain.

(3.4) Corollary. Let $S$ be an integral extension domain of a semi-local domain $R$. Then there exists a simple integral extension domain $S[e]$ of $S$ such that $c(S[e])=u c(S)$ and such that the following statements hold:

(3.4.1) For each integral extension domain $B$ of $S[e]$ and for each maximal ideal $P$ in $B$ such that either (i) or (ii) of (3.1.1) holds we have $u c(P)=c(P)=$ $u c\left(M^{\prime}\right)$ for some maximal ideal $M^{\prime}$ in $R^{\prime}$.

(3.4.2) If $R / M$ is infinite for each maximal ideal $M$ in $R$, then e can be chosen such that for each integral extension domain $B$ of $S[e]$ and for each maximal ideal $P$ in $B, u c(P)=c(P)=u c\left(N^{\prime}\right)$ for some maximal ideal $N^{\prime}$ in $S^{\prime}$.

Proof. By (3.1) there exists an integral extension domain $R[e]$ of $R$ such that $c(R[e])=u c(R)$. Then $u c(R)=c(R[e]) \subseteq c(S[e])$, by (2.3.4), and $c(S[e]) \subseteq u c(S[e])=u c(S)=u c(R)$, by (2.3.1) and (2.3.4), so $c(S[e])=$ $u c(S)$.

Statement (3.4.1) follows immediately from (3.1.1), since $R[e] \subseteq S[e] \subseteq B$.

For (3.4.2) it follows from (3.1.2) and the parenthetical statement in (2.5) that $u c(P)=c(P)=u c\left(M^{\prime}\right)$, where $M^{\prime}=P^{\prime} \cap R^{\prime}$ with $P^{\prime}$ a maximal ideal in $B^{\prime}$ that lies over $P$. Let $Q=P^{\prime} \cap S^{\prime}$ and let $U=S^{\prime}-Q$. Then it remains to show that $u c(Q)=u c\left(M^{\prime}\right)$. For this, $u c(Q)=u c\left(S^{\prime}[e]_{U}\right)$ by (2.3.4), and for each maximal ideal $Q^{\prime}$ in $S^{\prime}[e]_{U}$ we have $u c\left(Q^{\prime}\right)=c\left(Q^{\prime}\right)=u c\left(M^{\prime}\right)$, by (3.1.2) and the parenthetical statement in (2.5). Therefore by (2.3.3) we have $u c\left(M^{\prime}\right)=u c\left(S^{\prime}[e]_{U}\right)=u c(Q)$, as desired. Q.E.D.

The existence of a certain type of height one prime ideal in $R[X]$ is shown in (3.5). The ring $R\langle X\rangle$ in (3.5) is defined to be the quotient ring of $R[X]$ with respect to $S=R[X]-\cup\{N ; N$ is a maximal ideal in $R[X]$ such that $N \cap R$ is maximal \}. In [2], among other properties of $R\langle X\rangle$, it is noted that its maximal ideals are the ideals $N R\langle X\rangle$ with $N$ a maximal ideal in $R[X]$ 
such that $N \cap R$ is maximal, when $R$ is semi-local. This will be used in (3.5).

(3.5) Corollary. Let $R$ be a semi-local domain. Then there exists a height one prime ideal $K$ in $R\langle X\rangle$ such that $K$ contains a monic polynomial and such that $n \in c(R\langle X\rangle)$ if and only if there exists a maximal chain of prime ideals of length $n$ through $K$ in $R\langle X\rangle$.

Proof. It is known [5, (2.14.1) $\leftrightarrow(2.14 .5)]$ that

$$
c\left(R[X]_{N}\right)=\left\{m+1 ; m \in u c\left(R_{N \cap R}\right)\right\}
$$

for each maximal ideal $N$ in $R[X]$ such that $N \cap R$ is maximal. Therefore since the maximal ideals in $R\langle X\rangle$ are the extensions of such maximal ideals $N$ in $R[X]$, as noted above, it follows from (2.3.2) and (2.3.3) that $c(R\langle X\rangle)=$ $\{n+1 ; n \in u c(R)\}$ (since $R\langle X\rangle_{N R\langle X\rangle}=R[X]_{N}$ ). Therefore if $R[e]$ is as in (3.1), then the conclusion follows on letting $K=K^{\prime} R\langle X\rangle$, where $K^{\prime}=$ $\operatorname{Ker}(R[X] \rightarrow R[e])$. Q.E.D.

Let $R$ be a local domain such that there are no height one maximal ideals in $R^{\prime}$. If it can be shown that there exists an ideal $K$ as in (3.5) that is contained in only one maximal ideal in $R\langle X\rangle$, then it follows from (2.3.5) (and the property of $K$ ) that the equivalence of the Upper Conjecture noted following (3.2) holds (since

$$
R\langle X\rangle / K=R[X] /(K \cap R[X])
$$

and since $c(R\langle X\rangle)=\{n+1 ; n \in u c(R)\}$ as noted in the proof of (3.5)).

Our final result generalizes (3.1) in two ways: to the case when $R$ contains zero divisors, and to more general types of integral extension rings of $R$ than the ring $A$ in the proof of (3.1).

(3.6) Proposition. Let $R \subseteq A$ be quasi-semi-local rings such that $R$ is Noetherian, $A$ is integral over $R$, and minimal prime ideals in $A$ lie over minimal prime ideals in $R$. Then there exists a simple integral extension ring $R[e]$ of $R$ such that $c(A)=c(A[e])=c(R[e])$.

Proof. By (2.6.2) let $e$ be such that $A, A[e]$, and $R[e]$ all have the same number of maximal ideals and such that minimal prime ideals in $A[e]$ lie over minimal prime ideals in $A$. Then for each maximal ideal $Q$ in $R[e]$ there exist unique maximal ideals $P$ and $N$ in $A[e]$ and $A$, respectively, such that $A[e]_{P}$ is integral over $R[e]_{Q}$ and over $A_{N}$. Let $a \in N$ such that $a$ is not in any other maximal ideal in $A$ and let $p=N \cap R[a]$, so $A_{N}$ is integral over $R[a]_{p}$ as is $A[e]_{P}$. Now by hypothesis and the choice of $e$ it follows that minimal prime ideals in $A[e]_{P}$ lie over minimal prime ideals in $R[e]_{Q}$ and in $R[a]_{p}$ and that minimal prime ideals in $A_{N}$ lie over minimal prime ideals in $R[a]_{p}$. Therefore, by (2.3.5), $c(Q)=c(P)=c(p)$ and $c(N)=c(p)$. Therefore, $c(Q)=c(P)=$ $c(N)$, hence the conclusion follows from (2.3.2) and the one-to-one correspondences. Q.E.D.

Let $R$ be a semi-local domain. It would be of interest to know if there exists 
a quasi-semi-local integral extension domain $A$ of $R$ such that $c(A)=S$ whenever $S$ is a given subset of $u c(R)$ such that $c(R) \subseteq S$. It would also be of interest to know if there exists an integral extension domain $B$ of $R$ such that $B$ has exactly $n=\operatorname{card}(u c(R))$ maximal ideals and $c(B)=u c(R)$. (The first holds if the Upper Conjecture holds.) If either of these holds, then it also holds for some simple integral extension domain of $R$, by (3.6) and its proof.

\section{REFERENCES}

1. E. G. Houston and S. McAdam, Chains of primes in Noetherian rings, Indiana Univ. Math. J. 24 (1975), 741-753.

2. S. McAdam, On taut-level $R\langle X\rangle$, Duke Math. J. 42 (1975), 637-644.

3. M. Nagata, Local rings, Interscience Tracts no. 13, Interscience Publishers, New York, 1962.

4. L. J. Ratliff, Jr., Four notes on saturated chains of prime ideals, J. Algebra 39 (1976), 75-93.

5. L. J. Ratliff, Jr. and S. McAdam, Maximal chains of prime ideals in integral extension domains. I, Trans. Amer. Math. Soc. 224 (1976), 103-116.

6. L. J. Ratliff, Jr., Maximal chains of prime ideals in integral extension domains. II, Trans. Amer. Math. Soc. 224 (1976), 117-141.

7. , Maximal chains of prime ideals in integral extension domains. III, Illinois J. Math. (to appear).

8. Chain conjectures in ring theory, Lecture Notes in Math., vol. 647, Springer-Verlag, Berlin-Heidelberg-New York, 1978.

9. __ On maximal ideals and simple integral extension rings, preprint.

Department of Mathematics, University of California, Riverside, California 92521 